\title{
Perfect Difference Codes for Synchronous Fiber-Optic CDMA Communication Systems
}

\author{
Chi-Shun Weng and Jingshown $\mathrm{Wu}$
}

\begin{abstract}
In this paper, we introduce the perfect difference sets and propose a synchronous fiber-optic code-division multiple-access system (CDMA) using these sets to generate the signature codes. Two interesting properties of these codes are discussed: 1) any two different codes are cyclic-shifted with each other and 2) the cross correlation between any two different codes is exactly one. Thus, we may treat these codes as quasi-orthogonal codes. Using the first property of these codes, we can simplify design of the transmitter. In the receiver, we can use the second property to effectively eliminate the multiple-user interference (MUI) without reducing the number of usable codes. Based on the proposed transmitter and receiver, the system performance is derived. The numerical examples reveal that the proposed system can provide reliable communication even under heavy load. We believe that the proposed system using perfect difference codes outperforms any other synchronous fiber-optic CDMA systems.
\end{abstract}

Index Terms-Multiple-user interference (MUI), optical code division multiple access (CDMA), perfect difference set, spread spectrum.

\section{INTRODUCTION}

$\mathbf{R}$ ECENTLY, fiber-optic code-division multiple-access (CDMA) systems have attracted much attention. Some techniques have been proposed [1]-[6]. Because synchronous optical CDMA (SOCDMA) systems are more efficient to utilize the bandwidth than any asynchronous optical CDMA systems, many studies of SOCDMA systems have been reported in the literature [7]-[12]. There are several possible ways to achieve slot synchronization. One way is that we add a synchronizer node located at the star coupler [13]. This node broadcasts a pulse called the sync pulse periodically. Using the periodic pulses, the synchronization may be achieved among all the transmitters.

One of the major concerns of designing a direct-detection SOCDMA system is the multiple-user interference (MUI) because the performance of this system is usually interference limited. That is, there is an asymptotic error floor no matter how much power the receiver received. Unlike in the electric domain, the signature sequence in a direct-detection SOCDMA consists of unipolar $(0,1)$, sequences. As a result, there is no strict orthogonal codes under the constraint that the total number of codes is equal to the code length.

Manuscript received June 22, 2000; revised October 2, 2000. This work was supported by the National Science Council and Ministry of Education, Taiwan, R.O.C., under Grants NSC89-2215-E-002-012 and 89-E-FA06-2-4.

The authors are with the Department of Electrical Engineering and Graduate Institute of Communication Engineering, National Taiwan University, Taipei, Taiwan 10617, R.O.C.

Publisher Item Identifier S 0733-8724(01)01097-0.
With this constraint, the modified prime sequence codes [5], which are popular for SOCDMA systems, provide some desirable characteristics. If two codes are cyclic-shifted with each other, then the cross correlation between these two codes is zero. We may say that the two codes are orthogonal with each other and are in the same group. If two codes are not in the same group, i.e., not cyclic-shifted with each other, then the cross correlation is one. There are some reports of using these codes as the signature codes for the SOCDMA systems to reduce the MUI [7]-[12]. Generally speaking, we may classify these SOCDMA systems using the modified prime sequence codes into two categories. One is that the total number of usable codes is equal to the code length $p^{2}$, where $p$ is a prime [9]-[12]. The other is that the number of usable codes is less than $p^{2}$ [7], [8]. The performances in the first category are still MUI constraint and the bit error probability can not be reduced arbitrarily by increasing received optical power. On the other hand, the systems in the second category preserve the last code sequence in each group to cancel the MUI. It is effective to reduce the MUI and the error floor can be easily eliminated. However, these systems have the maximal number of users $p^{2}-p$ instead of $p^{2}$.

Another important issue for the SOCDMA systems is the architectures of the encoder in the transmitter and the decoder in the receiver because they will affect the power budget and reconfiguration time of the systems [14]. In this paper, we study the "perfect difference sets" and propose a new SOCDMA system using these sets as the signature code sequences instead of modified prime sequences [15]. The perfect difference codes have the following interesting characteristics:

1) any two distinct codes are cyclic-shifted with each other;

2) the cross correlation between any two distinct codes is exactly one.

However, the modified prime sequence codes have the cross correlation either one or zero. Although these codes are not strictly orthogonal, we may say that they are quasi-orthogonal. Using the first property, we propose a simple transmitter structure, in which the code sequence can be cyclic-shifted to get another transmitter. Using the second property, we also can easily design the receiver to remove the effect of the MUI. The system performance, with consideration of shot noise, thermal noise, avalanche photodiode (APD) bulk and surface leakage currents, is analytically formulated. The numerical examples show that the proposed system is very effective in eliminating the effect of the MUI. Moreover, the reliable communication $\left(P_{e} \leq 10^{-9}\right)$ of the proposed system is achievable even under heavy load.

The remainder of this paper is organized as follows. In Section II, we describe the perfect difference sets and the 
TABLE I

Some EXAmple of the Perfect Difference Sets

\begin{tabular}{|c|c|c|c|c|c|c|c|c|c|c|c|c|c|c|c|}
\hline$k$ & $v$ & \multicolumn{14}{|c|}{ perfect difference set } \\
\hline 3 & 7 & 0 & 1 & 3 & & & & & & & & & & & \\
\hline 4 & 13 & 0 & 1 & 3 & 9 & & & & & & & & & & \\
\hline 5 & 21 & 0 & 1 & 4 & 14 & 16 & & & & & & & & & \\
\hline 6 & 31 & 0 & 1 & 3 & 8 & 12 & 18 & & & & & & & & \\
\hline 8 & 57 & 0 & 1 & 3 & 13 & 32 & 36 & 43 & 52 & & & & & & \\
\hline 9 & 73 & 0 & 1 & 3 & 7 & 15 & 31 & 36 & 54 & 63 & & & & & \\
\hline 10 & 91 & 0 & 1 & 3 & 9 & 27 & 49 & 56 & 61 & 77 & 81 & & & & \\
\hline 12 & 133 & 0 & 1 & 3 & 12 & 20 & 34 & 38 & 81 & 88 & 94 & 104 & 109 & & \\
\hline 14 & 183 & 0 & 1 & 3 & 16 & 23 & 28 & 42 & 76 & 82 & 86 & 119 & 137 & 154 & 175 \\
\hline
\end{tabular}

sufficient condition to construct these sets. The characteristics of the perfect difference codes are also discussed. The feasible transmitter and receiver structures based on these codes are described in Section III. In Section IV, we analyze the system performance and present the bit error probability as functions of received optical power, noise power, code length, and the number of simultaneous users. Section $\mathrm{V}$ shows the numerical results and performance comparisons with other SOCDMA systems. Finally, we give the conclusion in Section VI.

\section{The Perfect Difference SeT}

Definition: Let $W$ be the $v$-set of the integers $0,1, \ldots, v-1$ modulo $v$. A set $D=\left\{d_{1}, d_{2}, \ldots, d_{k}\right\}$ is a $k$-subset of $W$. For every $a \neq 0(\bmod v)$, there are exactly $\lambda$ ordered pairs $\left(d_{i}, d_{j}\right), i \neq j$, such that

$$
d_{i}-d_{j} \equiv a(\bmod v) .
$$

A set $D$ fulfilling these requirements is called a perfect difference set or $(v, k, \lambda)$-cyclic difference set modulo $v$. The $v$ cyclic-shifted sets $D_{\tau}=\left\{d_{1}-\tau, d_{2}-\tau, \ldots, d_{k}-\tau\right\}$ modulo $v, \tau=0,1, \ldots,(v-1)$, all have the same property, where $D_{0}=D$ and each $D_{i} \cap D_{j}$ for $i \neq j$ is a $\lambda$-subset of $W$. Since there are $k(k-1)$ pairs and the total number for $a \neq 0(\bmod v)$ is $(v-1)$, the relation among $v, k, \lambda$ is

$$
\lambda=\frac{k(k-1)}{v-1} .
$$

Because of the rigid property, arbitrary values of $v, k$, and $\lambda$ do not in general yield a perfect difference set. However, a special type of perfect difference sets is the $(v, k, \lambda=1)$-cyclic difference set. The existence of the $(v, k=q+1, \lambda=1)$-cyclic difference set, where $q$ is any power of any prime, has been proved and constructed by Singer [15]. Because it has a good property with minimum number of overlap, $\lambda=1$, between two cyclic-shifted sets, we take the advantage of this cyclic difference set to construct the signature codes. For brevity, we name such set as cyclic difference set through this paper. More detailed information about the perfect difference sets can be found in [16] and [17]. Some cyclic difference sets are presented in Table I. Notice that there is not only one set for each $k=q+1$ [15]. Actually, the number of distinct cyclic difference sets, which are not cyclic-shifted with one another, for each $k=q+1$ is even, since each cyclic difference set can be paired with its inverse.

We can construct $v$ perfect difference codes $C_{\tau}=$ $\left\{c_{\tau, 0}, c_{\tau, 1}, \ldots, c_{\tau, i}, \ldots, c_{\tau,(v-1)}\right\}, \tau \in W$, based on the cyclic difference set with the rule

$$
c_{\tau, i}= \begin{cases}1, & \text { for } i \in D_{\tau} \\ 0, & \text { otherwise. }\end{cases}
$$

The code weight, code length, and total number of codes are $k, v$, and $v$, respectively, where $v=k^{2}-k+1$. An example for $k=5$ is presented in Table II. From the property of the cyclic difference set, the correlation $\Phi_{x, y}$ between any two codes $C_{x}$ and $C_{y}$ can be expressed as

$$
\Phi_{x, y}= \begin{cases}k, & \text { if } x=y \\ 1, & \text { if } x \neq y\end{cases}
$$

Although these perfect difference codes are not strictly orthogonal codes, we may say that they are quasi-orthogonal codes because of the unity cross correlation between two distinct codes. Notice that the correlation in (4) is the same as that in the extended modified prime sequence codes [18]. However, the cyclic-shifted property of perfect difference codes makes the design of a tunable transmitter or a tunable receiver simpler.

\section{SYSTEM DESCRIPTION}

\section{A. Transmitter Structure}

For each subscriber, it is assigned a unique code sequence. When User $x$ wants to send a bit to User $y$ over a bit duration $T_{b}$, it sends $k$ optical pulses correspondent to User $y$ 's code sequence if the data bit is 1 , otherwise, no pulses are sent. Using the characteristic of the cyclic difference codes, we propose an all-optical tunable SOCDMA transmitter structure as shown in Fig. 1. The input optical pulse is split into $k$ branches with equal power. In the $i$ th branch, there is a fixed optical delay line with delay $d_{i} \cdot T_{c}$, where $d_{i} \in D_{0}$ and $T_{c}$ represent a chip duration given by

$$
T_{c}=\frac{T_{b}}{v} .
$$

Thus, if there is no any relative delay in the following tunable delay lines, the output optical pulses are correspondent with the first code $C_{0}$. In order to cyclic-shift $C_{0}$ to form another code $C_{j}$, the delay selector between the splitter and combiner, which 
TABLE II

THE PERFECT DifFERENCE CODES FOR $k=5$

\begin{tabular}{|c|c|c|c|c|c|c|c|c|c|}
\hline$j$ & \multicolumn{7}{|c|}{ Cyclic Difference Sets } & \multicolumn{2}{|c|}{ Perfect Difference Codes } \\
\hline 0 & $D_{0}$ & $=\{$ & 0 & 1 & 4 & 14 & 16 & $C_{0}$ & $=110010000000001010000$ \\
\hline 1 & $D_{1}$ & $=\{$ & 20 & 0 & 3 & 13 & 15 & $C_{1}$ & $=100100000000010100001$ \\
\hline 2 & $D_{2}$ & $=\{$ & 19 & 20 & 2 & 12 & 14 & $C_{2}$ & $=001000000000101000011$ \\
\hline 3 & $D_{3}$ & $=\{$ & 18 & 19 & 1 & 11 & 13 & $C_{3}$ & $=010000000001010000110$ \\
\hline 4 & $D_{4}$ & $=\{$ & 17 & 18 & 0 & 10 & 12 & $C_{4}$ & $=100000000010100001100$ \\
\hline 5 & $D_{5}$ & $=\{$ & 16 & 17 & 20 & 9 & 11 & $C_{5}$ & $=000000000101000011001$ \\
\hline 6 & $D_{6}$ & $=\{$ & 15 & 16 & 19 & 8 & 10 & $C_{6}$ & $=000000001010000110010$ \\
\hline 7 & $D_{7}$ & $=\{$ & 14 & 15 & 18 & 7 & 9 & $C_{7}$ & $=000000010100001100100$ \\
\hline 8 & $D_{8}$ & $=\{$ & 13 & 14 & 17 & 6 & 8 & $C_{8}$ & $=000000101000011001000$ \\
\hline 9 & $D_{9}$ & $=\{$ & 12 & 13 & 16 & 5 & 7 & $C_{9}$ & $=000001010000110010000$ \\
\hline 10 & $D_{10}$ & $=\{$ & 11 & 12 & 15 & 4 & 6 & $C_{10}$ & 01100100000 \\
\hline 11 & $D_{11}$ & $=\{$ & 10 & 11 & 14 & 3 & 5 & $C_{11}$ & $=000101000011001000000$ \\
\hline 12 & $D_{12}$ & $=\{$ & 9 & 10 & 13 & 2 & 4 & $C_{12}$ & $=001010000110010000000$ \\
\hline 13 & $D_{13}$ & $=\{$ & 8 & 9 & 12 & 1 & 3 & $C_{13}$ & $=010100001100100000000$ \\
\hline 14 & $D_{14}$ & $=\{$ & 7 & 8 & 11 & 0 & 2 & $C_{14}$ & $=101000011001000000000$ \\
\hline 15 & $D_{15}$ & $=\{$ & 6 & 7 & 10 & 20 & 1 & $C_{15}$ & $=010000110010000000001$ \\
\hline 16 & $D_{16}$ & $=\{$ & 5 & 6 & 9 & 19 & 0 & $C_{16}$ & $=100001100100000000010$ \\
\hline 17 & $D_{17}$ & $=\{$ & 4 & 5 & 8 & 18 & 20 & $C_{17}$ & $=000011001000000000101$ \\
\hline 18 & $D_{18}$ & $=\{$ & 3 & 4 & 7 & 17 & 19 & $C_{18}$ & $=000110010000000001010$ \\
\hline 19 & $D_{19}$ & $=\{$ & 2 & 3 & 6 & 16 & 18 & $C_{19}$ & $=001100100000000010100$ \\
\hline 20 & $D_{20}$ & $=\{$ & 1 & 2 & 5 & 15 & 17 & $C_{20}$ & $=011001000000000101000$ \\
\hline
\end{tabular}

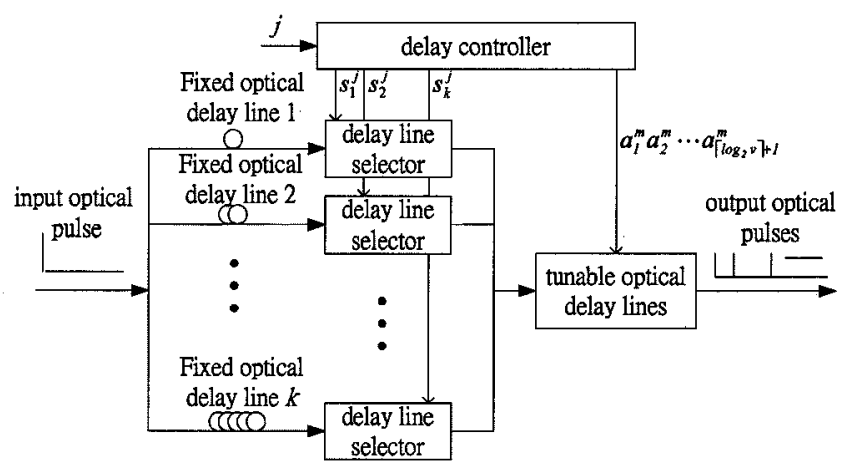

Fig. 1. Tunable transmitter structure.

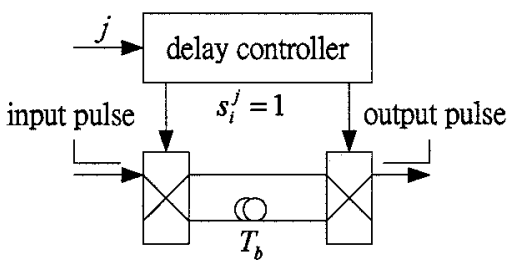

Fig. 2. Delay line selector in the $i$ th branch.

contains two $2 \times 2$ optical switches and optical delay lines as shown in Fig. 2, is needed. The delay in the lower optical delay line is a bit duration longer than that in the upper one. When the two switches are both set in the bar state by $s_{i}^{j}=0$, then the input pulse is no relative delay. If they are in the cross state by $s_{i}^{j}=1$, then one bit delay is introduced. The $s_{i}^{j}$, s are controlled by the delay controller and can be expressed as

$$
s_{i}^{j}= \begin{cases}1, & \text { if } j>d_{i} \\ 0, & \text { otherwise. }\end{cases}
$$

Therefore, the output $k$ pulses of the optical power combiner are correspondent to code $C_{j}$. Although the output pulses match to the code $C_{j}$, it is not the desired pattern because of losing synchronization with other users after the delay selectors. Hence, we need tunable optical delay lines, whose structure is shown in Fig. 3 [19], to delay $(v-j) \cdot T_{c}$ to restore the synchronization. For the sake of delay time from 0 to $v-1$ chip duration, there are $\left\lceil\log _{2} v\right\rceil+1$ stages of $2 \times 2$ switches, where $\lceil t\rceil$ denotes the smallest integer not less than $t$. And the $i$ th stage may introduce $2^{\left\lceil\log _{2} v\right\rceil-i} \cdot T_{c}$ delay, $i \in\left\{1,2, \ldots,\left\lceil\log _{2} v\right\rceil\right\}$. If it needs to delay $m \cdot T_{c}$, the delay controller selects control sequence $a_{1}^{m} a_{2}^{m} \ldots a_{\left\lceil\log _{2} v\right\rceil+1}^{m}$ appropriately to control the $\left\lceil\log _{2} v\right\rceil+1$ stages such that the total delay is equal to $m \cdot T_{c}$. The method to select the control sequence is as follows. First, it converts $m$ into $\left\lceil\log _{2} v\right\rceil$ binary sequence $b_{1}^{m} b_{2}^{m} \ldots b_{\left\lceil\log _{2} v\right\rceil}^{m}$ and pads two zero bits $b_{0}^{m}=0$ and $b_{\left\lceil\log _{2} v\right\rceil+1}^{m}=0$ before and after the binary sequence, respectively, to form the new binary sequence $b_{0}^{m} b_{1}^{m} b_{2}^{m} \ldots b_{\left\lceil\log _{2} v\right\rceil}^{m} b_{\left\lceil\log _{2} v\right\rceil+1}^{m}$. Then, let

$$
a_{i}^{m}=b_{i-1}^{m} \oplus b_{i}^{m}, \quad \text { for } i=1,2, \ldots,\left\lceil\log _{2} v\right\rceil+1
$$

where $\oplus$ means the Exclusive-OR operation and the control sequence $a_{1}^{m} a_{2}^{m} \ldots a_{\left\lceil\log _{2} v\right\rceil+1}^{m}$ is the desired one. Some examples of $k=5$ are presented in Table III. For suitable design, the subnanosecond reconfiguration time of the tunable optical delay lines may be feasible [19].

Another important concern on designing a SOCDMA system is the beating of the optical carriers. If the lasers from different transmitters simultaneously emit on the same wavelength it may destroy the signals in all channels. To reduce this effect, we may use a broadband light source or a polorization scrambler at the end of a transmitter to reduce the beating [20]. 


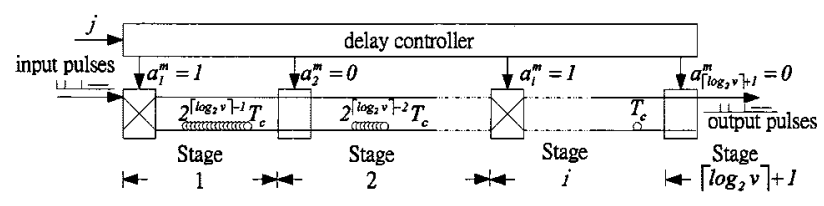

Fig. 3. Tunable optical delay lines.

\section{B. Receiver Structure}

As well as the transmitter, we can use the properties of the cyclic difference codes to structure a simple receiver. Unlike the tunable transmitter, the receiver is fixed in the proposed system as shown in Fig. 4. If it needs a tunable receiver, we also can apply the design principle of the tunable transmitter mentioned. Similar to the techniques proposed in [7]-[10], we use the second branch to eliminate the MUI. The received signal power per pulse, $P$, is split into two branches with unequal power, $P_{1}$ and $P_{2}$ with respective to the first and the second branches. The first branch has an optical correlator, an APD, an integrator, and a sampler. The optical correlator contains $k$ tap delay lines correspondent to the desired code sequence such that the $k$ chips are delayed to the last chip duration. The correlator output is then photodetected, integrated over the last chip duration, and sampled at the end of the bit duration. The output $Y_{1}$ of the sampler contains the desired signal, interference from other users, and noise. The second branch has an APD, an integrator, a sampler, and a divider. After integrated over a bit duration and sampled, the output signal $Y_{2}$ is then divided by $r$, where $r$ is a constant and its optimal value only depends on the code weight $k$. The relation between $r$ and $k$ will be described and the performances with and without a divider will be presented later. After subtracting $Y_{2} / r$ from $Y_{1}$, the output signal $Y$ is then fed into the ON-OFF keying (OOK) demodulator. If $Y$ is less than the constant threshold value of the OOK demodulator the output bit is 0 , otherwise is 1 .

\section{Performance Analysis}

We assume that the number of simultaneous (active) users is $N$. To express the state of code $C_{j}, j \in W$, we define a random variable $z_{j}$ as

$$
z_{j}= \begin{cases}1, & \text { if code } C_{j} \text { belongs to an active user } \\ 0, & \text { otherwise. }\end{cases}
$$

Thus, the number of users who sent the data bit 1 within a bit time can be written as

$$
N_{1}=\sum_{j=0}^{v-1} b_{j} z_{j}
$$

where $b_{j} \in\{0,1\}$ denotes the binary data bit. Notice that $N_{1}$ is a random variable. Without loss of generality, we assume the first user with the assigned code $C_{0}$ is the desired user and $b_{0}$ is the desired data bit.

The average photon arrival rate $\lambda_{1}$ per pulse at the input of the optical correlator in the first branch is given by

$$
\lambda_{1}=\frac{\eta P_{1}}{h f}
$$

TABLE III

THE CONTROL SEQUENCE FOR $k=5$

\begin{tabular}{cccc}
\hline \hline $\begin{array}{c}\text { User } \\
j\end{array}$ & delay & binary bits & control sequence \\
& $m$ & $b_{0}^{m} b_{1}^{m} b_{2}^{m} b_{3}^{m} b_{4}^{m} b_{5}^{m} b_{6}^{m}$ & $a_{1}^{m} a_{2}^{m} a_{3}^{m} a_{4}^{m} a_{5}^{m} a_{6}^{m}$ \\
\hline 0 & 0 & 0000000 & 000000 \\
\hline 1 & 20 & 0101000 & 111100 \\
\hline 3 & 17 & 0100010 & 110011 \\
\hline 20 & 1 & 0000010 & 000011 \\
\hline \hline
\end{tabular}

where

$\eta \quad$ APD quantum efficiency;

$h \quad$ Plank's constant;

$f \quad$ optical frequency.

Given $N_{1}$ and the desired bit $b_{0}=1$, the conditional probability density function of the output $Y_{1}$ after the sampler in the first branch can be expressed as [6]

$$
P_{Y_{1}}\left(y_{1} \mid N_{1}, b_{0}=1\right)=\frac{1}{\sqrt{2 \pi \sigma_{y_{1}}^{2}}} e^{-\left(y_{1}-\mu_{y_{1}}\right)^{2} / 2 \sigma_{y_{1}}^{2}}
$$

where the mean of $Y_{1}, \mu_{y_{1}}$, can be expressed as

$$
\mu_{y_{1}}=G T_{c}\left[\left(\frac{k+N_{1}-1}{k}\right) \lambda_{1}+I_{b} / e\right]+T_{c} I_{s} / e .
$$

Here, $G$ is the average APD gain, $e$ is the electron charge, $I_{b} / e$ is the contribution of the APD bulk leakage current to the APD output, $I_{s}$ is the APD surface leakage current, and the variance of $Y_{1}, \sigma_{y_{1}}^{2}$, can be written as

$$
\sigma_{y_{1}}^{2}=G^{2} F_{e} T_{c}\left[\left(\frac{k+N_{1}-1}{k}\right) \lambda_{1}+I_{b} / e\right]+T_{c} I_{s} / e+\sigma_{t h}^{2}
$$

where $\sigma_{t h}^{2}$ is the variance of thermal noise and $F_{e}$ is the excess noise factor given by

$$
F_{e}=k_{\mathrm{eff}} G+(2-1 / G)\left(1-k_{\mathrm{eff}}\right) .
$$

Here, $k_{\text {eff }}$ is the APD effective ionization ratio.

$\sigma_{t h}^{2}$ is given by

$$
\sigma_{t h}^{2}=2 k_{B} T_{r} T_{c} /\left(e^{2} R_{L}\right)
$$

where

$k_{B} \quad$ Boltzmann's constant;

$T_{r} \quad$ receiver noise temperature;

$R_{L} \quad$ receiver load resistance.

Similarly, given $N_{1}$ and $b_{0}=0$, the conditional probability density function of $Y_{1}$ is

$$
P_{Y_{1}}\left(y_{1} \mid N_{1}, b_{0}=0\right)=\frac{1}{\sqrt{2 \pi \sigma^{\prime 2}}} e^{-\left(y_{1}-\mu_{y_{1}}^{\prime}\right)^{2} / 2 \sigma_{y_{1}}^{\prime 2}}
$$

where the mean of $Y_{1}, \mu_{y_{1}}^{\prime}$, can be expressed as

$$
\mu_{y_{1}}^{\prime}=G T_{c}\left[\left(\frac{N_{1}}{k}\right) \lambda_{1}+I_{b} / e\right]+T_{c} I_{s} / e
$$

and the variance of $Y_{1}, \sigma_{y_{1}}^{\prime 2}$, can be written as

$$
\sigma_{y_{1}}^{\prime 2}=G^{2} F_{e} T_{c}\left[\left(\frac{N_{1}}{k}\right) \lambda_{1}+I_{b} / e\right]+T_{c} I_{s} / e+\sigma_{t h}^{2} .
$$




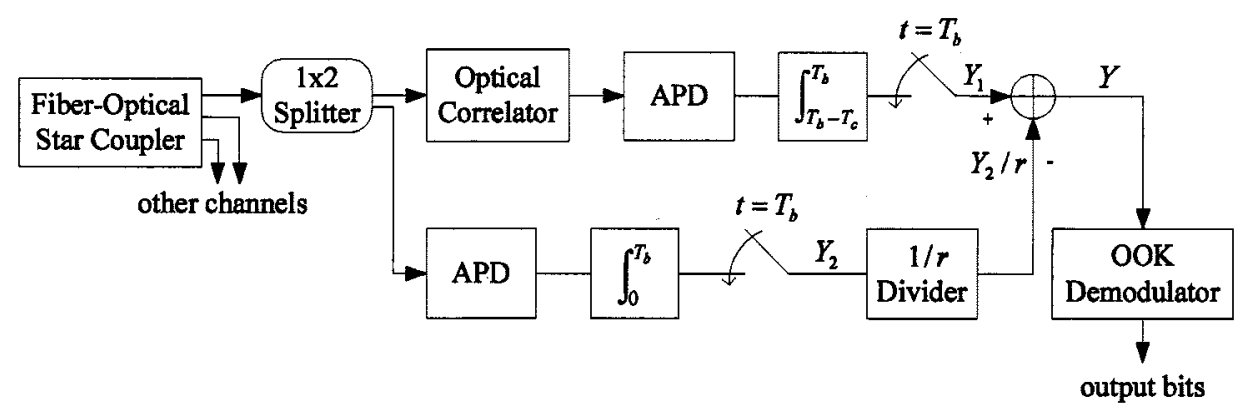

Fig. 4. The fixed receiver structure.

TABLE IV

LINK PARAMETERS

\begin{tabular}{lll}
\hline \hline Name & Symbol & Value \\
\hline Light wavelength & & $1.3 \mu m$ \\
\hline $\begin{array}{l}\text { APD } \\
\text { Quantum efficiency }\end{array}$ & $\eta$ & 0.6 \\
\hline APD gain & $G$ & 100 \\
\hline $\begin{array}{l}\text { APD effective } \\
\text { ionization ratio }\end{array}$ & $k_{e f f}$ & 0.02 \\
\hline $\begin{array}{l}\text { APD bulk } \\
\text { leakage current }\end{array}$ & $I_{b}$ & $0.1 \mathrm{nA}$ \\
\hline $\begin{array}{l}\text { APD surface } \\
\text { leakage current }\end{array}$ & $I_{s}$ & $10 \mathrm{nA}$ \\
\hline chip duration & $T_{c}$ & $0.1 \mathrm{~ns}$ \\
\hline $\begin{array}{l}\text { bit rate } \\
\frac{1}{T_{b}}=\frac{1}{v T_{c}}\end{array}$ & $300 \mathrm{k}$ \\
\hline $\begin{array}{l}\text { Receiver noise } \\
\text { temperature }\end{array}$ & $T_{r}$ & $1030 \Omega$ \\
\hline Receiver load resistor & $R_{L}$ & \\
\hline \hline
\end{tabular}

In the second branch, the average photon arrival rate $\lambda_{2}$ per pulse at the input of the APD is given by

$$
\lambda_{2}=\frac{\eta P_{2}}{h f} .
$$

Given $N_{1}$, the conditional probability density function of the output $Y_{2}$ after the sampler in the second branch can be expressed as

$$
P_{Y_{2}}\left(y_{2} \mid N_{1}\right)=\frac{1}{\sqrt{2 \pi \sigma_{y_{2}}^{2}}} e^{-\left(y_{2}-\mu_{y_{2}}\right)^{2} / 2 \sigma_{y_{2}}^{2}}
$$

where the mean of $Y_{2}, \mu_{y_{2}}$, can be expressed as

$$
\mu_{y_{2}}=G T_{c}\left[N_{1} k \lambda_{2}+v I_{b} / e\right]+v T_{c} I_{s} / e
$$

and the variance $\sigma_{y_{2}}^{2}$ is given by

$$
\sigma_{y_{2}}^{2}=G^{2} F_{e} T_{c}\left[N_{1} k \lambda_{2}+v I_{b} / e\right]+v\left(T_{c} I_{s} / e+\sigma_{t h}^{2}\right)
$$

respectively.

After subtracting the second signal $Y_{2} / r$ from the first signal $Y_{1}$, we obtain the result signal at the input of the OOK demodulator as

$$
Y=Y_{1}-\frac{1}{r} Y_{2}
$$

The mean of $Y$ is

$$
E(Y)=\left\{\begin{array}{rlrl}
G T_{c} & {\left[\left(\frac{k+N_{1}-1}{k}\right) \lambda_{1}-\frac{N_{1} k \lambda_{2}}{r}\right.} & \\
& \left.+\left(1-\frac{v}{r}\right) I_{b} / e\right] \\
+ & \left(1-\frac{v}{r}\right) T_{c} I_{s} / e, & \\
G T_{c} & {\left[\left(\frac{N_{1}}{k}\right) \lambda_{1}-\frac{N_{1} k \lambda_{2}}{r}\right.} & \\
& \left.+\left(1-\frac{v}{r}\right) I_{b} / e\right] & \\
+\left(1-\frac{v}{r}\right) T_{c} I_{s} / e, & \text { if } b_{0}=1
\end{array}\right.
$$

To cancel the MUI, we set

$$
\frac{N_{1} \lambda_{1}}{k}=\frac{N_{1} k \lambda_{2}}{r} .
$$

That is,

$$
\begin{aligned}
P_{1} & =\frac{k^{2}}{r} P_{2} \\
& =\frac{k^{2} / r}{k^{2} / r+1} P .
\end{aligned}
$$

Therefore, the mean and variance of $Y$ are

$E(Y)= \begin{cases}G T_{c}\left[\left(\frac{k-1}{k}\right) \lambda_{1}+\left(1-\frac{v}{r}\right) I_{b} / e\right] & \\ +\left(1-\frac{v}{r}\right) T_{c} I_{s} / e, & \text { if } b_{0}=1 \\ G T_{c}\left[\left(1-\frac{v}{r}\right) I_{b} / e\right]+\left(1-\frac{v}{r}\right) T_{c} I_{s} / e, & \text { if } b_{0}=0\end{cases}$

and

$$
\operatorname{Var}(Y)= \begin{cases}\sigma_{Y}^{2}=\sigma_{y_{1}}^{2}+\frac{1}{r^{2}} \sigma_{y_{2}}^{2}, & \text { if } b_{0}=1 \\ \sigma_{Y}^{\prime 2}=\sigma_{y_{1}}^{\prime 2}+\frac{1}{r^{2}} \sigma_{y_{2}}^{2}, & \text { if } b_{0}=0\end{cases}
$$

respectively. 
Hence, the constant threshold value of the OOK demodulator is set as

$\theta=G T_{c}\left[\left(\frac{k-1}{2 k}\right) \lambda_{1}+\left(1-\frac{v}{r}\right) I_{b} / e\right]+\left(1-\frac{v}{r}\right) T_{c} I_{s} / e$.

The bit error probability given the number of simultaneous users $N=n$ can be derived as follows:

$$
\begin{aligned}
& P_{r}(\text { bit error } \mid N=n) \\
& =P_{r}\left(Y \geq \theta \mid N=n, b_{0}=0\right) \cdot P_{r}\left(b_{0}=0\right) \\
& \quad+P_{r}\left(Y<\theta \mid N=n, b_{0}=1\right) \cdot P_{r}\left(b_{0}=1\right) \\
& =\sum_{m=0}^{n-1} P_{r}\left(Y \geq \theta \mid N_{1}=m, b_{0}=0\right) \\
& \quad \cdot P_{r}\left(N_{1}=m \mid N=n, b_{0}=0\right) \cdot P_{r}\left(b_{0}=0\right) \\
& \quad+\sum_{m=1}^{n} P_{r}\left(Y<\theta \mid N_{1}=m, b_{0}=1\right) \\
& \quad \cdot P_{r}\left(N_{1}=m \mid N=n, b_{0}=1\right) \cdot P_{r}\left(b_{0}=1\right)
\end{aligned}
$$

where

$$
\begin{aligned}
& P_{r}\left(Y \geq \theta \mid N_{1}=m, b_{0}=0\right)=\frac{1}{2} \operatorname{erfc}\left(\frac{G T_{c}\left(\frac{k-1}{2 k}\right) \lambda_{1}}{\sqrt{2 \sigma_{Y}^{\prime 2}}}\right) \\
& P_{r}\left(Y<\theta \mid N_{1}=m, b_{0}=1\right)=\frac{1}{2} \operatorname{erfc}\left(\frac{G T_{c}\left(\frac{k-1}{2 k}\right) \lambda_{1}}{\sqrt{2 \sigma_{Y}^{2}}}\right) \\
& P_{r}\left(N_{1}=m \mid N=n, b_{0}=0\right)=\left(\begin{array}{c}
n-1 \\
m
\end{array}\right) \cdot \frac{1}{2^{n-1}} \\
& P_{r}\left(N_{1}=m \mid N=n, b_{0}=1\right)=\left(\begin{array}{c}
n-1 \\
m-1
\end{array}\right) \cdot \frac{1}{2^{n-1}} .
\end{aligned}
$$

Here, $\operatorname{erfc}(\cdot)$ stands for the complementary error function, defined as

$$
\operatorname{erfc}(z)=\frac{2}{\sqrt{\pi}} \int_{z}^{\infty} \exp \left(-u^{2}\right) d u
$$

and it is assumed that $P_{r}\left(b_{0}=0\right)=P_{r}\left(b_{0}=1\right)=1 / 2$.

To analyze the optimal value of $r$, we take the maximum noise into consideration. In other words, the number of users who sent data bit 1 is the same as the maximum number of subscribers, that is, $N_{1}=v$. In this case, we assume that the shot noise is dominant and other noise is negligible. Hence, the variance of $Y$ becomes

$$
\begin{aligned}
\operatorname{Var}(Y) & =\sigma_{y_{1}}^{2}+\frac{1}{r^{2}} \sigma_{y_{2}}^{2} \\
& =G^{2} F_{e} T_{c}\left(k+\frac{v}{k r}\right) \lambda_{1} .
\end{aligned}
$$

The effective $\operatorname{SNR}(r)$ can be written as

$$
\begin{aligned}
\operatorname{SNR}(r) & =\frac{\left[G T_{c}\left(\frac{k-1}{k}\right) \lambda_{1}\right]^{2}}{G^{2} F_{e} T_{c}\left(k+\frac{v}{k r}\right) \lambda_{1}} \\
& =\left(\frac{k^{2} / r}{k^{2} / r+1}\right) \cdot \frac{1}{\left(k+\frac{v}{k r}\right)} \cdot \frac{T_{c} \lambda}{F_{e}} \cdot\left(\frac{k-1}{k}\right)^{2} \\
& =\Psi(r) \cdot \frac{T_{c} \lambda}{F_{e}} \cdot\left(\frac{k-1}{k}\right)^{2}
\end{aligned}
$$

where

$$
\Psi(r)=\left(\frac{k^{2} / r}{k^{2} / r+1}\right) \cdot \frac{1}{\left(k+\frac{v}{k r}\right)}
$$

and $\lambda$ is the average photon arrival rate per pulse before the splitter and is given by

$$
\lambda=\frac{\eta P}{h f} .
$$

Maximizing $\operatorname{SNR}(r)$ is the same as maximizing the $\Psi(r)$. And the maximum value of $\Psi(r)$ is obtained under

$$
r=\sqrt{k^{2}-k+1}
$$

\section{NUMERICAL RESULTS}

In this section, we present the numerical results of the systems using the perfect difference codes. Since the performances of the two SOCDMA systems [8], [7] (which is the same as Canceler 1 in [8]) are not MUI constraint, we compare here the performances of three SOCDMA systems: 1) the system with interference estimator [8]; 2) the proposed system without a divider; and 3) the proposed system with an optimal divider. Because the Canceler 2 has the best performance in the systems with interference estimators [8], we only take the Canceler 2 into consideration. Furthermore, we assume that there is no error on estimating the interference in that system. Thus, the performances of the systems with interference estimators are the lower bound. Some common parameters and their values are listed in Table IV. Other parameter values yield similar results.

The relation between $\Psi(r)$ and $r$ for various values of $k$ is shown in Fig. 5. The optimal value of $r$ is around 12. For $k=17$, the value of $P_{1}$ is almost equal to $0.95 P$ according to (26). In other words, the most received power is assigned to the first branch. On the other hand, if we do not use a divider in the second branch to optimize the performance, i.e., $r=1$, the value of $P_{1}$ is almost equal to $P$. It seems that the performance of the system without the divider is better than that using a divider due to a little larger power in the first branch. However, the noise power of $Y$ in the system without a divider is much larger than that using a divider due to that the noise power contributed from the second branch of the former system is much larger than that of the latter one according to (28). As a result, the performance of the system without a divider is about 2.42 $\mathrm{dB}$ worse than that of the system using an optimal divider. The 


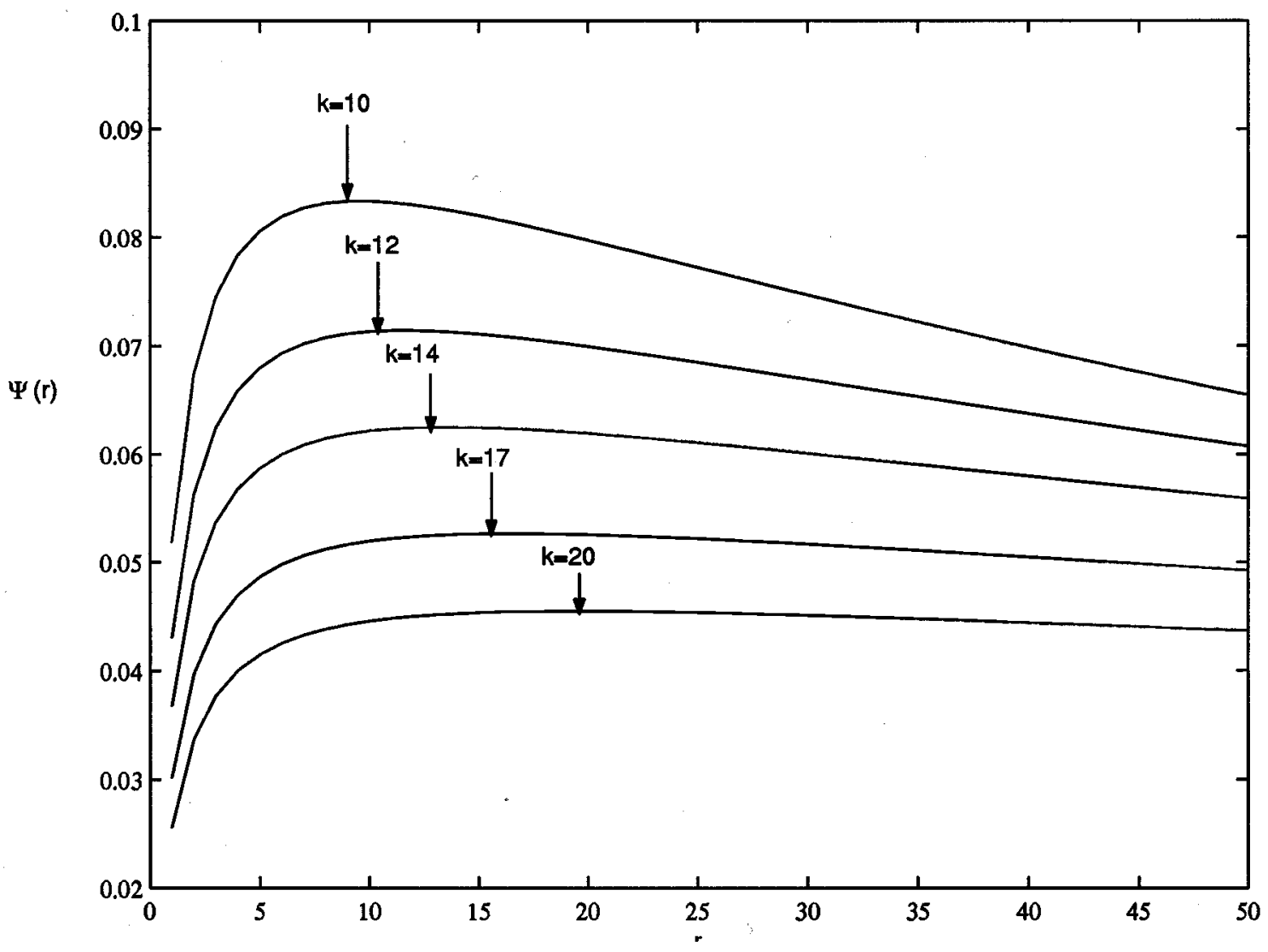

Fig. 5. Relation between $\Psi(r)$ and $r$.

figure also shows that the curves come down with the value of $k$ increases due to the raise of shot noise.

Fig. 6 shows the performances of three SOCDMA systems under the same received power per pulse $P=10 \mu \mathrm{W}$. Firstly, we compare the performances between our proposed systems with and without an optimal divider. The same as discussed above, the performance of the proposed system without a divider is much worse than that of the proposed system using an optimal divider. On the other hand, when the number of simultaneous users increases the performances are getting worse due to the increment of shot noise. Moreover, the bit error probability is smaller for larger code weight $k$ under the same number of simultaneous users. This is because that each undesired user who sends the data bit 1 contributed $1 / k$ optical pulse into the last chip duration at the output of optical correlator. And it also induces the correspondent shot noise after the photodetector. Therefore, the larger code weight results in the smaller shot noise and bit error probability. Secondly, we compare the performances between the systems with interference estimators and the proposed systems with an optimal divider. Because the code length for the modified prime sequence codes is always the square of a prime there is no equal code length between any modified prime sequence codes and perfect difference codes. Thus, we compare the performance between the code weights $p=11$ (code length $=121)$ and $p=13$ (code length $=169)$ for modified prime sequence codes and the code weight $k=12$ (code length $=133$ ) and $k=14$ (code length $=183$ ) for perfect difference codes. The numerical results show that the perfor- mances of the systems using interference estimators under code weight $p=11$ and $p=13$ are worse than those of the proposed systems with an optimal divider under code weight $k=12$ and $k=14$. By the way, the performance of the former systems is also worse than that of the latter system under the same code weight $p=17$ (code length $=289$ ) or $k=17$ (code length $=273$ ). This is because that the power in the first branch of the proposed systems is almost two times larger than that of the systems with interference estimators. Moreover, the noise power contributed from the second branch of the proposed system is suppressed by the optimal divider. As a result, the effective SNR of the proposed system with an optimal divider is larger than that of the systems with interference estimators. Note that we do not need to keep any code to estimate the interference of the proposed system but the last code in each group is never assigned to any user and is reserved for the MUI estimation at the receiving end of the systems with interference estimators [8]. Thus, the proposed systems are more efficient to utilize the codes than the systems with interference estimators.

Under the maximum number of simultaneous users $N=v$ in the proposed systems or $N=p^{2}-p$ in the systems with interference estimators, the bit error probability versus the received power $P$ is shown in Fig. 7. For the received power is $16 \mu \mathrm{W}$ and $k \leq 17$, the bit error probability of the system with the optimal divider is smaller than $10^{-9}$. The results also show that the performance of the proposed systems without a divider or the systems with interference estimators is worse than that of the proposed systems with the optimal divider. Moreover, the 


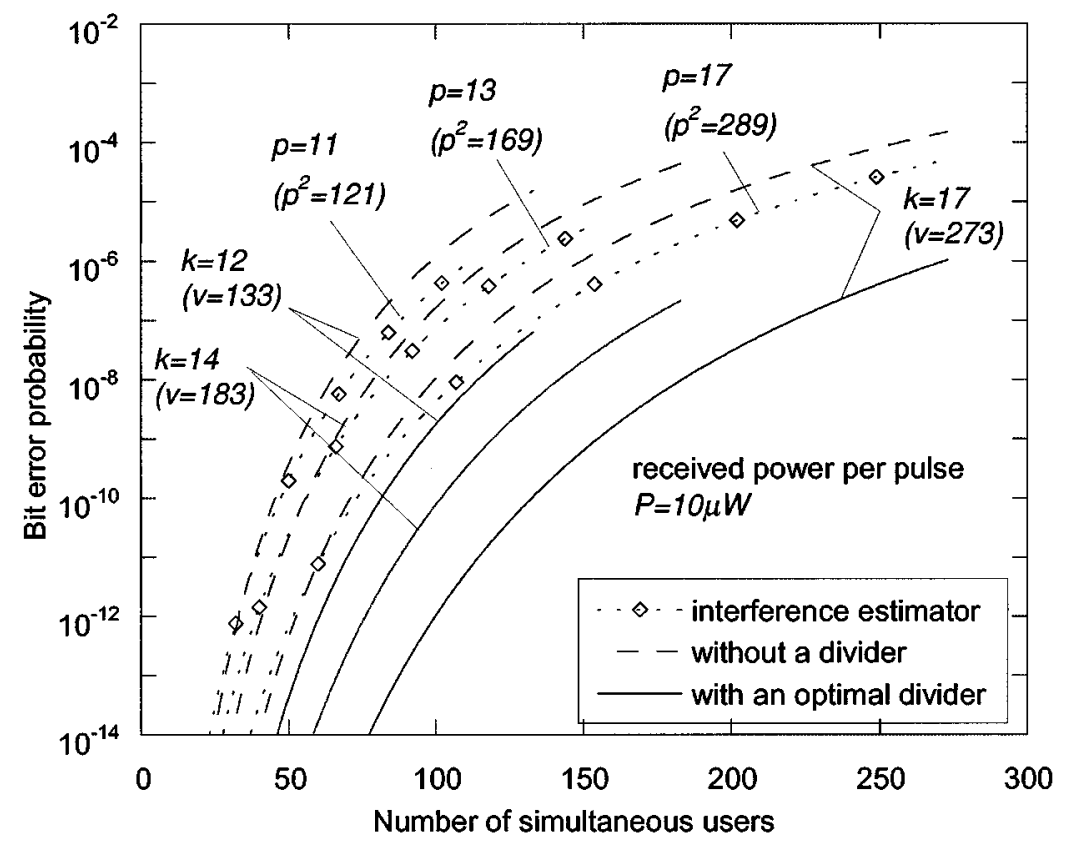

Fig. 6. Bit error probability comparisons under the same received power per pulse $P=10 \mu \mathrm{W}$.

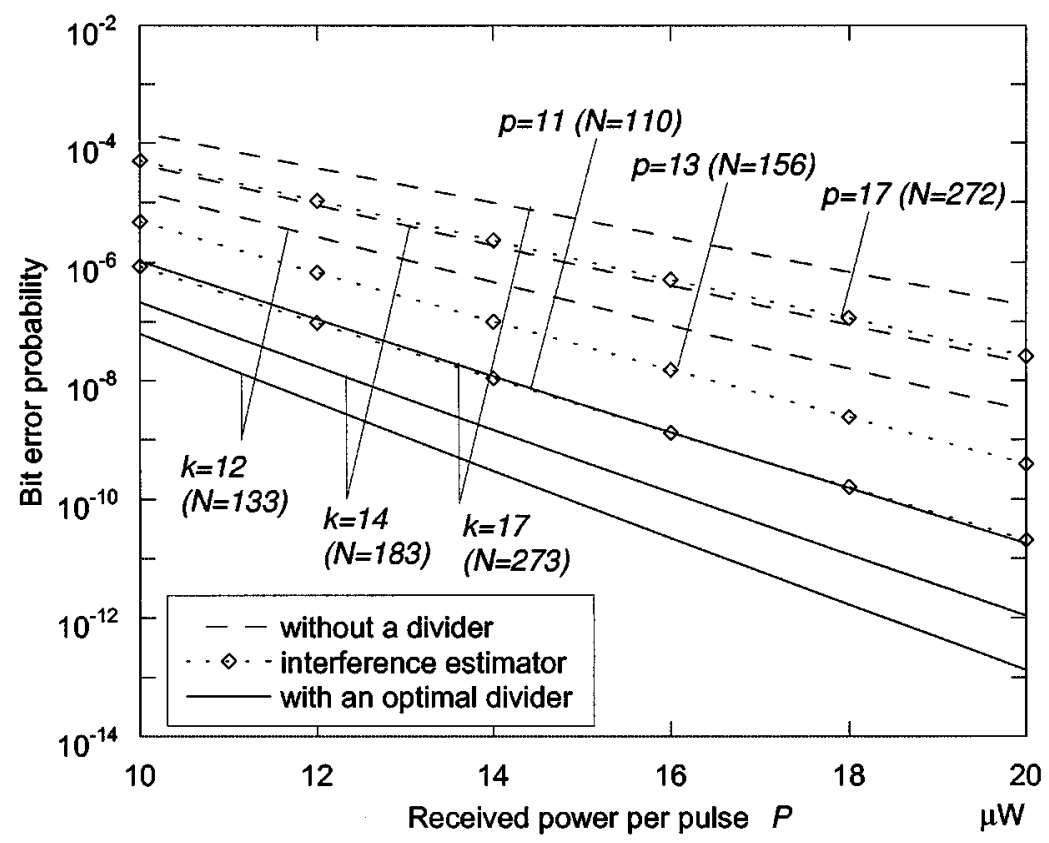

Fig. 7. Bit error probability comparisons under different code weights and full load.

larger the code weight has, the worse the performance is due to more shot noise. Hence, it reveals that the bit error probability is going down with the increment of received power. In other words, the error floor can be completely removed and the performance of the system is no longer interference limited.

\section{CONCLUSION}

The application of the perfect difference sets in the SOCDMA systems is investigated first time. We also propose a new SOCDMA system using these sets as the signature codes. Based on the cyclic-shifted and unity cross correlation properties of these codes, we propose the transmitter and receiver structures. An optimal divider is used on the receiver to enhance the system performance. The bit error probability is derived analytically. The numerical results, with consideration of shot noise, thermal noise, APD bulk, and surface leakage currents, show that it is effective to eliminate the MUI and the asymptotic error floor which appears in the SOCDMA systems with modified prime sequence codes. Moreover, the proposed system can provide reliable communication even under heavy load without reducing the number of usable codes. It is believed 
that the proposed system performs better than other SOCDMA systems.

\section{ACKNOWLEDGMENT}

The authors would like to thank the anonymous reviewers for valuable suggestions.

\section{REFERENCES}

[1] P. R. Prucnal, M. A. Santoro, and T. R. Fan, "Spread spectrum fiber-optic local area network using optical processing," J. Lightwave Technol., vol. LT-4, pp. 547-554, May 1986.

[2] F. R. K. Chung, J. A. Salehi, and V. L. Wei, "Optical orthogonal codes: Design, analysis, and applications," IEEE Trans. Inform. Theory, vol. 35, pp. 595-604, May 1989.

[3] J. A. Salehi, "Code division multiple-access techniques in optical fiber networks-Part I: Fundamental priciples," IEEE Trans. Commun., vol. 37, pp. 824-833, Aug. 1989.

[4] J. A. Salehi and C. A. Brackett, "Code division multiple-access techniques in optical fiber networks-Part II: Systems performance analysis," IEEE Trans. Commun., vol. 37, pp. 834-842, Aug. 1989.

[5] W. C. Kwong, P. A. Perrier, and P. R. Prucnal, "Performance comparison of asynchronous and synchronous code-division multiple access techniques for fiber-optic local area networks," IEEE Trans. Commun., vol. 39, no. 11, pp. 1625-1634, Nov. 1991.

[6] H. M. Kwon, "Optical orthogonal code-division multiple-acess system-Part I: APD noise and thermal noise," IEEE Trans. Commun., vol. 42, July 1994.

[7] H. H. Shalaby, M. A. Mangoud, and S. E. El-Khamy, "A new interference cancellation technique for synchronous CDMA communication systems using modified prime codes," in Proc. 2nd IEEE Symp. Computers and Communications, July 1997, pp. 556-560.

[8] H. M. H. Shalaby, "Synchronous fiber-optic CDMA systems with interference estimators," J. Lightwave Technol., vol. 17, pp. 2268-2275, Nov. 1999.

[9] — , "Cochannel interference reduction in optical PPM-CDMA systems," IEEE Trans. Commun., vol. 46, pp. 799-805, June 1998.
[10] C.-L. Lin and J. Wu, "A synchronous fiber-optic CDMA system using adaptive optical hardlimiter," J. Lightwave Technol., vol. 16, pp. 1393-1403, Aug. 1998.

[11] T. Ohtsuki, K. Sato, I. Sasase, and S. Mori, "Direct-detection optical synchronous CDMA systems with double optical hard-limiters using modified prime sequence codes," J. Lightwave Technol., vol. 14, pp. 1879-1887, Sept. 1996.

[12] C.-L. Lin and J. Wu, "Channel interference reduction using random manchester codes for both synchronous and asynchronous fiber-optic CDMA systems," J. Lightwave Technol., vol. 18, pp. 26-33, Jan. 2000.

[13] R. Ramaswami and K. N. Sivarajan, Optical networks: A practical perspective. San Mateo, CA: Morgan Kaufmann, 1998.

[14] W. C. Kwong and P. R. Prucnal, "All-serial coding architecture for ultrafast optical code-division multiple-access," in Proc. ICC '93, vol. 1, May 1993, pp. 552-556.

[15] J. Singer, "A theorem in finite projective geometry and some applications to number theory," Trans. Amer. Math. Soc., vol. 43, pp. 377-385, 1938.

[16] M. Hall Jr., Combinatorial Theory. London, U.K.: Blaisdell, 1967.

[17] H. J. Ryser, Combinatorial Mathematics. New York: Wiley, 1963.

[18] C.-S. Weng and J. Wu, "Extended modified prime sequence codes for synchronous optical CDMA communication system," in Proc. OECC'2000, Chiba, Japan, July 2000, pp. 188-189.

[19] P. R. Prucnal, M. F. Krol, and J. L. Stacy, "Demonstration of a rapidly tunable opical time-division-multiple-access coder," IEEE Photon. Technol. Lett., vol. 3, pp. 170-172, Feb. 1991.

[20] F. Heismann, "Compact electro-optic polarization scramblers for optically amplified lightwave systems," J. Lightwave Technol., vol. 14, pp. 1801-1814, Aug. 1996.

Chi-Shun Weng, photograph and biography not available at the time of publication.

Jingshown Wu, photograph and biography not available at the time of publication. 